Ansicht über die Constitution der Diglycolsäure entwickelt. - Ich wollte hier nur die Gelegenheit ergreifen, um an einem Beispiel, in welchem von Kolbe und mir zu verschiedenen Zeiten und ganz unabhängig von einander dieselbe Idee fast mit den gleichen Worten ausgesprochen ist, zu zeigen, dass zwischen der Richtung derjenigen Chemiker, welche auf die Atomigkeitslehre basirend Stracturformeln, welche bis auf die Bindung der Atome eingehen, zu entwickeln suchen und den Anschauungen Kolbe's kein so wesentlicher Unterschied besteht, wie man dieses so oft angenommen findet.

Froiburg, d. 28. Febr. 1871.

\title{
Ueber die Structurformeln und die Lehre von der Bindung der Atome;
}

\section{von \\ H. Kolbe.}

Am Schlnss der vorstehenden Abhandlung hat Claus die Meinung auggesprochen, dass meine Vorstellungen über die Constitution der chemischen Verbindungen nicht wesentlich verschieden seien von den Ansichten derjenigen Chemiker, welche auf die Atomigkeitslehre basirend Structurformeln entwickeln, die bis auf die Bindung der einzelnen Atome eingehen.

Ich bin entgegengesetzter Meinung, und habe zunächst berichtigend zu bemerken, dass auch ich auf der Atomigkeitslehre fusse, ich glaube sogar behaupten zu dürfen, dass nächst Frankland, dem wir eigentlich die Atomigkeitslehre verdanken, wenn or auch nicht gerade diesen Ausdruck gebraucht hat, ich es gewesen bin, der auf dieser Grundlage, aus der früheren Hypothese der gepaarten Radioale heraus, zuerst die Ideen über die chemische Constitution der fetten Säuren, Alkohole, Aldehyde etc. entwickelt habe, welche später von den Chemikern 
stillschweigend adoptirt, und weiterhin durch die Strueturformeln resp. die denselben zu Grunde liegenden Vorstellungen, meiner Ansicht nach, - es möge mir das Wort nicht iibel genommen werden - corrumpirt sind.

Nach meiner Auffassung gleisht die Constitution einer chemischen Verbindung derjenigen eines gut organisirten constitutionellen Staates mit cinem Oberhaupte und mehreren ihm näher oder entfernter stehenden untergeordneten Gliedern, welche so organisirt sind, dass darin an Stelle des einzelnen Individuums eine aus verschiedenen Individuen bestehende Gruppe von gleichem Range fungiren kann. Als Beispiel mögen die Homologen des Methylalkohols dienen.

Von den zwei Kohlenstoffatomen des Aethylalkohols steht das eine im Range über den anderen, und fungirt, um in dem Vergleiche fortzufshren, als Oberhaupt der Verbindung. Es ist dies dasselbe Kohlenstoffatom, welchem im Methylalkohol die vier Glieder H, H, H und OH unterstellt sind. Wenn überhaupt darüber ein Zweifel sein kann, dass im Aethylalkohol das zweite Kohlenstoffatom für die ganze Verbindung eine ganz andere und zwar untergeordnetere Bedeutung hat, als jenes dominirende erste Kohlenstoffatom, so braucht man nur zu bedenken, dass wenn das zweite Kohlenstoffatom in Gemeinschaft mit 3 At. Wasserstoff als Methyl an Stelle von 1 At. Wasserstoff in jenen Methylalkohol substituirend eingetreten ist, der chemische Charakter dieses letzteren dadurch wenig alterirt wird, dass mithin jenes zweite Kohlenstoffatom für das Bestehen einer Verbindung von den Eigenschaften des Alkobols nicht nothwendig, das erstere dominirende Kohienstorfatom dagegen nnentbehrlich ist. Selbstverständlich spielt im Propylalkohol das dritte Kohlenstoffatom, welchea mit 3 At. Wasserstoff als Methyl in das Methyl des Aethylradicals für 1 At. Wasserstoff sich einfügt, eine noch untergeordnetere Rolle.

Um diese Einschachtelungen von Methyl an Stelle von Wasserstoff, und zugleich den verschiedenen Rang der einzelnen Glieder im Aethylalkohol, Propylalkohol und Butyl- 
und die Lehre von der Bindung der Atome. 129 alkohol symbolisch auszudrücken, wähle ich beispielsweise folgende Formeln, in denen del Rang der einzelnen Grösseu durch die Umrahmung, wie auch durch vertehiedene Grösse der Schrift bezeichnet ist.

$\mathrm{H}_{3}$ / C OH - Methylalkohol.

$\left.\frac{\mathrm{H}_{2} \mathrm{C}}{\mathrm{H}_{2}}\right\} \mathbf{C ~ O H}$ - Aethylalkohol.
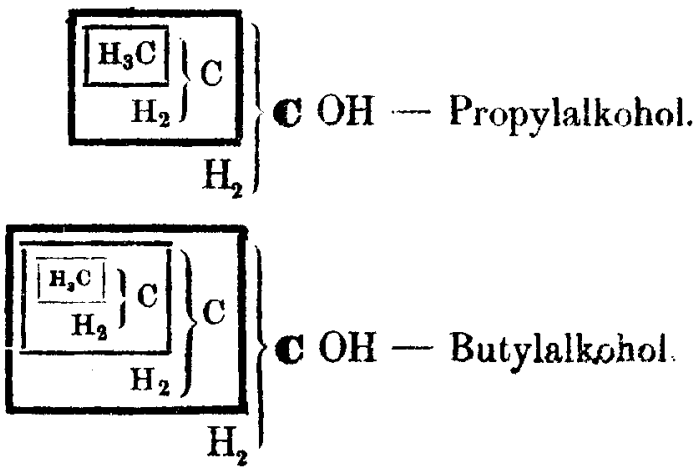

Wem das Methyl des Aethylalkohols als wirklich existirendes Substitut von 1 At. Warserstoff gilt, der muss, meine ich, mit mir weiter daraus folgern, dass nicht der Kohlenstoff dieses Methyls direct an dem anderen Koblenstoffatom des Aethylalkohols hängt, sondern dass die Methylgruppe als einheitliches Ganzes (natürlich in Folge ibrer Zusammensetzung einwerthig) mit dem Hauptkohlenstoffatom des Alkohols ebenso verbunden zu denken ist, wie das einfache Wasserstoffatom, dessen Stelle es vertritt.

Diejenigen Chemiker, welche bis auf die Bindung der einzelnen Atome eingebende Structurformeln schreiben, statuiren, falls ich recht unterrichtet bin, und wie auch in ihren Structurformeln ausgesprochen liegt, keine Rangunterschiede der gleichnamigen Bestandtheile einer Verbindung; deshalk müssen ihnen manche einfache $\mathrm{Zu}$ sammensetzungs-Verhältnisse unverständlich bleỉen. Ein Beispiel möge diese Behauptung erläutern.

Journ. f. prakt, Chemis [8] Bd. 8 . 
Es wird, wenn man darauf ausgeht, nicht schwer sein, ein Propylchlorid darzustellen, in dessen untergeordnetstem Methylatom noch 1 At. Wasserstoff durch Chlor vertreten ist, also von der empirischen Zusammensetzung: $\mathrm{C}_{3} \mathrm{H}_{6} \mathrm{Cl}_{2}$, eine Verbindung, deren rationelle Zusammensetzung ich durch die Formel: $\left.\left.{ }^{\mathrm{Cl}}{ }^{\mathrm{H}_{2}} \underset{\mathrm{H}_{2}}{\mathrm{C}}\right\} \underset{\mathrm{C}}{\mathrm{C}}\right\} \mathbf{C ~ C l}$ ausdrücken würde.

Ungewohnt mit Structurformeln zu operiren, glaube ich doch nicht zu irren, wenn ich meine, dass die Structurchemiker die Zusammensetzung dieser Verbindung durch folgendes Schema veranschaulichen:<smiles>ClCCCl</smiles>

In diesem Schema unterscheidet sich das untere der drei Kohlenstoffatome durch Nichts von dem oberen, und man köunte daher glauben, dass die mit diesen beiden Kohlenstoffatomen in unmittelbarer Verbindung gedachten beiden Chloratome gloiche Bedeutung, gleiche Functionen haben, dass mithin das eine Chlor eben so leicht wie das andere etwa durch Hydroxyl sich muisse ersetzen lassen.

Wenn nun jenes gecnlorte Propylchlorid auch noch nicht dargestellt ist, so kann man doch mit Gewissheit behaupten, dass dasselbe beim Erhitzen mit wässeriger Kalilauge (analog dem gechlorten Propionsäurechlorid) nur ein Atom Chlor mit Hydroxyl vertauschen wird, und dass das andere unangegriffen bleibt, dass mithin ein gechlorter Propylalkohol resultirt.

Die Structurformel reicht also in diesem wie in anderen Fällen nicht aus, um dem Leser die Zusammensetzungsweise und Natur der Verbindung deutlich zu machen, wie 
und die Lehre von der Bindung der Atome. 131

denn überhaupt die Structurformel, welche nichts aussagt von dem dominirenden Haupte in der chemischen Verbindung, einem Satze gleicht, dem das Subject fehlt.

Auf die Gefahr hin, damit anzustossen, oder Missfallen zu erregen, will ich hier mein Urtheil über die neuere Lehre von der Bindung der Elemente, wie sie sich in den Structurformeln abspiegelt, off̣en aussprechen.

Dje Gesohichte der theoretischen Chemie während der letzten 30 Jahre hat gelehrt, dass, so oft man die Lehre von dem wirklichen Vorhandensein zusammengesetzter Radicale in den organischen Verbindungen verlassen hat, man jedesmal anf Abwege gerathen ist: so Gerhardt, als er die Bestrebungen Anderer, über die chemische Constitution der Verbindungen überhaupt sich. Rechenschaft zu geben, verhöhnte und den erassesten Empirismus predigte, sodann kurze Zeit hernach derselbe, als er seinen Irrthum gewahr wurde, aber ihn weder eingestehen, noch auch zur Radicaltheorie zurückkehren mochte, und in der Typentheorie den Chemikern ein unfruchtbures Schema zur nackten Gruppirung der Verbindungen darbot, neuerdinge die Chemiker, welche die wirkliche Existenz zusammengesetzter Radicale in den organischen Verbindungen und deren verschiedene Functionen in denselben verleugnend sich begnügen, die Bindung der elementaren Atome nach ihren Valenzen zu erklären, und symmetrisch gebaute Structurformeln zu schreiben.

Wenn ich diese neuere Art, die Constitution oder Structur der chemisohen Verbindungen zu erklären, einen Irrweg nenne, so bin ich darauf gefasst, dass mir entgegnet wird, die grosse Mehrzahl der Chemiker folge ja eben dieser Richtung, und es sei doch wohl nicht anzunehmen, dass diese Alle einen Irrweg einseblagen. Diese Argumentation ist indessen hinfällig. Ich erinnere daran, dass vor nicht gar langer Zeit - noch sind seitdem nicht 10 Jahre verflossen - , die ganz grosse Mehrzahl der Chemiker der Typentheorie huldigte, und dass ich damals 
fast allein dieselbe bekümpfend ${ }^{1}$ ) die jetzt wohl allgemein getheilte Ansicht verfocht, dass die Chemie höhere wissenschaftliche Ziele zu erstreben habe, als die T'ypentheorie mit ihrem Classifications-Formular ermöglichte.

Ich erblicke mich gegenwärtig wieder in der nämlichen Lage wie damals. Fast allein bekämpfe ich die Lehre von der Bindung der Atome in dem Sinne, wie sie sich in den Structurformeln ausspricht, und ich hege die feste Ueberzeugung, dass wie heute fast Niemand mehr von Typen und Typentheorie als überwundenem Standpunkte spricht, man in wenigen Jahren allgemein auch einsehen gelernt haben wird, dass bei Untersuchung der chemischen Constitution der organischen Verbindung mehr zu leisten ist, als hübsche Structurformeln zu bilden.

Ich erachte es immer als ein bedenkliches und verdüchtiges Zeichen für eine Lehre, wenn dieselbe schwierige Fragen so leicht beantwortet, dass ganz junge unerfahrene Chemiker darüber mitreden und aburtheilen können. Das eben war es, was seiner Zeit der Typentheorie unter den ganz jungen Chemikern enthusiastische Freunde erwarb, denn es bedurfte nur weniger Sachkenntniss, um die Verbindungen zu formuliren und gehörig zu classificiren, und das ist es auch jetzt wieder, was der einseitigen Lehre von der Bindung der Atome so schnell Eingang verschafft.

Wie leicht es die Bindungslehre macht, die chemische Constitution complicirt zusammengesetzter Verbindungen, selbst solcher, die noch sehr wenig studirt sind, durch Structurformeln zu interpretiren, dafür entnehme ich unter hundert andern Beispielen den Beweis einer Abhandlang

1) Ich finde hio und $d a$ dit Meinung ausgesprochen, ich habe die Principien der Typentheorie ebenfalls adoptirt gehabt. Dieser Irrthum ist sielleicht dadurch entstanden, dass ich von den vier Typen jener Typen. theorie den einen, nämlich das Ammoniak als realen Typus an. erkannt oder vielmehr noch früher, ehe terhardt seine Theorie auf. stellte, nach $H$ ofmann und auf Grund seiner Entdenkungen als die typische Grundlage aller organischer Ammoniake angeaehen habe. 
aus dem eben erschienenen Februibrheft der Annalen der Chemie (Bd. 157), wovon Seite 206 zufällig gerade vor mir aulgeschlagen liegt. Fin junger Chemiker, von dem meines Wissens chemische Untersuchungen nicht weiter vorliegen, beginnt seine wissenschaftlichen Leistungen mit „Studien über die Constitution des Diamylens", und proponirt für dasselbe wie auch für ein von ihm dargestelltes Oxydationsproduct von der Zusammensetzung $\mathrm{C}_{10} \mathrm{H}_{20} \mathrm{O}$, trotzdem dass über die chemische Natur dieses Körpers so gut wie Nichts bekannt ist, elegante Structurformeln, welche den Leser mit Staunen erfüllen müssten über den tiefen Einblick, den die Bindungalehre in den chemischen Organismus jener Verbindungen zu thun ermöglicht, wenn derselbe nicht sogleich begriffe, dass das Alles nichts als glänzende Seifenblasen sind.

Ich lasse hier je eine der wahrhaft unbegreiflichen Structurformeln folgen, welche in jener Abhandlung S. 206 für das Diamylen und dessen Oxydationsproduct rorgeschlagen und discutirt sind:

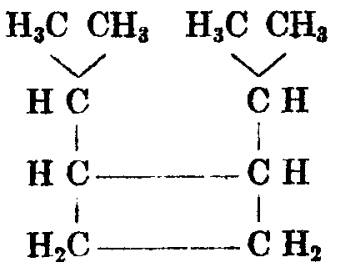

Formel für das Diamylen.

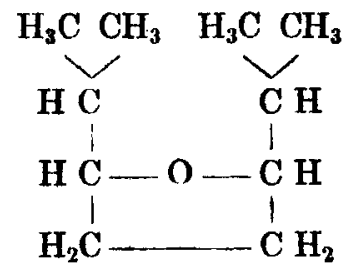

Formel für das Oxydationsproduct: $\mathrm{C}_{10} \mathrm{H}_{20} \mathrm{O}$.

Es liegt mir durchaus fern, dem Verfasser jener Arbeit einen Vorwurf machen zu wollen, es ist wie gesagt Zufall, dass ich gerade diese Abhandlung herausgegriffen habe, um an einem Beispiele darzuthun, wie bestechend für junge Chemiker die Lehre von der Bindung der Atome sein muss, da sie auch Unerfahrene befähigt, subtile Fragen zu discutiren und darüber zu philosophiren. Der reifere Chemiker weiss und hat es hundert Mal selbst erfahren, dass die Natur sich ihre Geheimnisse so leicht nicht ablauschen lässt. 
Eine grosse Sckwäche der Lehre von der Bindung der Atome besteht noch darin, dass sie ihre Begründer und Anhänger, zumal wenn dieselben dabei noch den Satz aufrecht erhalten wissen wollen, dass jedes Element immer nur eine bestimmte Süttigungseapacitat besitzt, zu mancherlei willkührlichen Annahmen nöthigt. Als solche erachte ich unter anderen dib, dass in Benzol die sechs Kohlenstoffatome abwechselnd mit je einer und mit je zwei Affinitäten an einander gekettet sein sollen. Viel plausibeler und weniger unwahrscheinlich würde mir, wenn ich überhaupt eine dieser Auffassungen gelten lassen könnte, die von Claus aufgestellte Hypotbese sein, dass die sechs Kohlenstoffatome des Benzols nach Art der in beistehendem Schema sich ausdrückenden Vorstellung

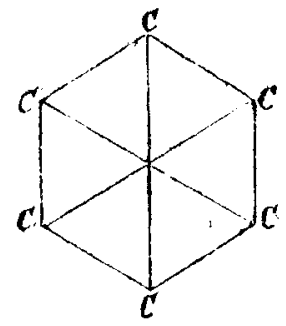

an einander gefesselt sind.

Nicht minder willkührlich und im hohen Grade unwahrscheinlich ist mir die Annuhme, dass die beiden Sauerstoffatome der Chinone durch eine Valenz mit einander selbst verbunden seien, und daher zusammen gleich dem einfachen Sauerstoffatome als divalente Atomgruppe fungiren.

Wie kommt es, muss man hier fragen, dass bei den Oxydationsprocessen, wo Chinone entstehen, ein solches unter sich gebundenes divalentes Sauerstoffpaar und nicht wie bei der Oxydation anderer Körper ein einfaches Sauerstoffatom eintritt? und warum begegnen wir einem solchen Vorgange bei Oxydationen nicht häufiger?

Würde man, wo zwei Sauerstoftatome derselben Werth haben und denselben Effect hervorbringen, wie eins von 
beiden allein, nicht erwarten dürfen, dass das eine überschüssige Sauerstoffatom sich leicht abtrennen und so eine gleiohfalls gesättigte Verbindung minus 1 At. Sauerstoff sich darstellen lasse?

Diese und ähnliche Fragen sind moines Erachtens gewichtig genug, um nach allen Seiten hin reiflich erwogen zu werden, ehe man sich $\iota \mathrm{u}$ der sehr gewagten Hypothese entschliesst, dass zwei Sauerstoffatome in Folge gegenseitiger Bindung (unnöthiger Weise) die Arbeit eines einzigen Sauerstoffatoms verrichten.

Ich bin hier auf den Einwand gefasst, dass eine andere einfachere Erklärung von der Constitution des Chinons nicht möglich sei. Es mag sein, dass die Bindungsund Verkettungslehre keine bessere Interpretation zu geben vermag, ich selbst traue mir darüber kein Urtheil zu, aber ich meine, dass man von einem anderen Standpunkte aus die Constitution des Chinons sehr wohl interpretiren kann. ohne zu der gewagten Hypothese von der Divalenz zweier Sauerstoffatome Zufincht zu nehmen.

In einer vor etwa zwei Jahren veröffentlichten Broohüre „über die chemische Constitation der Kohlenwasserstofie" habe ich die Hypothese aufgestellt, dass das Benzol eine den Triaminen ähnliche Constitution habe, nämlich ein Tricarbol sei, $d$. h. eine Verbindung von 3 At. Kohlenstoff mit 3 At. der dreiwerthigen Gruppe (CH) "' und 3 At. Wasserstoff, und dass das Phenol an Stelle eines dieser drei Wasserstoffatome Hydroxyl enthalte, wie folgende Formeln verdeutlichen mögen:

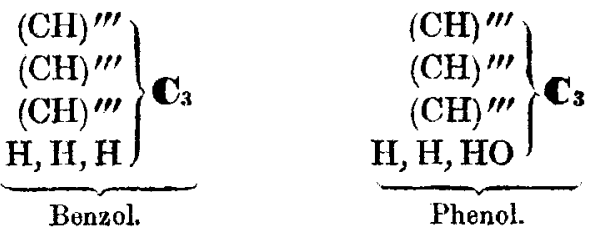

Den noch nicht unmittelbar effectuirten Uebergang des Pherols in Chinon oder wenigstens die Beziehungen beider Verbindungen kann man einfach so auffassen, dass zwei 
136 Kolbe: Ueber die Structurformeln etc.

Atome Wasserstoff von zweien der dreiwerthigen Radicale (CH) durch 1 At. Sauerstoff ersetzt werden in ähnlicher Weise, wie 1 At. Sauerstoff in zwei Molekülen Essigsüure 2 At. Wasserstoff substituirt, wenn Diglycolsäure entsteht. Bei der soweit gleich verlaufenden Bildung des Chloranils werden die übrigen vier Wasserstoffatome des Phenols zu gleicher Zeit durch Chlor ersetzt. Folgende drei Formeln mögen das veranschaulichen:

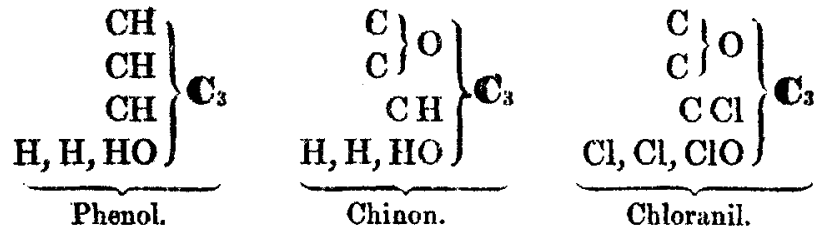

Ich lasse es dahin gestellt sein, ob die eben ausgesprochene, durch vorstehende Formeln illustrirte Idee von der Constitution der betreffenden Verbindungen die richtige ist, ich wollte damit nur zeigen, dass man die $\mathrm{Zu}$ sammensetzung der Chinone sehr wohl interpretiren kann, ohne zu der gewagten Annahme greifen zu müssen, dass darin die zwei Ssuerstoffatome mit je einer Valenz an einander gekettet seien, und so den Werth von bloss einem Sauerstoffatom hahen.

Mir obigen Auseinandersetzungen habe ich bezweckt, die Chemiker davon zu überzeugen, dass ich bei den Untersuchungen über die chemische Constitution der organischen Verbindungen von anderen Grundsätzen ausgehe, als die Chemiker, welche mit der Bindungslehre Structurformeln bauen, und dass zwischen beiden Auffassungen und Principien ein sehr wesentlicher Unterschied besteht. 